\title{
Change Detection in Dual Polarization Sentinel-1 Data with Wilks' Lambda
}

\author{
Nielsen, Allan Aasbjerg
}

Published in:

IEEE Geoscience and Remote Sensing Letters

Link to article, DOI:

10.1109/LGRS.2021.3068516

Publication date:

2021

Document Version

Peer reviewed version

Link back to DTU Orbit

Citation (APA):

Nielsen, A. A. (2021). Change Detection in Dual Polarization Sentinel-1 Data with Wilks' Lambda. IEEE Geoscience and Remote Sensing Letters, 19. https://doi.org/10.1109/LGRS.2021.3068516

\section{General rights}

Copyright and moral rights for the publications made accessible in the public portal are retained by the authors and/or other copyright owners and it is a condition of accessing publications that users recognise and abide by the legal requirements associated with these rights.

- Users may download and print one copy of any publication from the public portal for the purpose of private study or research.

- You may not further distribute the material or use it for any profit-making activity or commercial gain

- You may freely distribute the URL identifying the publication in the public portal

If you believe that this document breaches copyright please contact us providing details, and we will remove access to the work immediately and investigate your claim. 


\title{
Change Detection in Dual Polarization Sentinel-1 Data with Wilks' Lambda
}

\author{
Allan Aasbjerg Nielsen
}

\begin{abstract}
When the covariance matrix formulation is used for multi-look polarimetric synthetic aperture radar (SAR) data, Wilks' Lambda can be used for change detection between acquisitions at two time points. We briefly describe the theory for dual polarization, diagonal only data. A case study illustrates the technique on Sentinel-1 C-band data covering the international Frankfurt Airport. We successfully detect change including direction of change in the sense that Wilks' Lambda shows whether radar signal increases or decreases over time.
\end{abstract}

Index Terms-Complex covariance matrices, generation of complex Wishart distributed matrices, Beta distribution approximation, dual polarization, remote sensing change detection, Sentinel-1.

\section{INTRODUCTION}

This paper presents a method for change detection in bitemporal, multi-look, dual polarization, diagonal only, synthetic aperture radar (SAR) data in the covariance matrix representation. Many authors have worked with this subject (including the fully polarimetric case), see for example [1][7]. In [4], [6] we deal not only with bi-temporal but with truly multi-temporal polarimetric SAR data.

The method presented here uses Wilks' Lambda [8] which is an established quantity in multivariate statistics. We use it to determine whether radar signal as measured by the determinant of the covariance matrix has increased or decreased over time. We also give an associated p-value in a Beta distribution approximation to establish whether statistically significant change has occurred over time. In this paper the use of the Beta distribution approximation is based on simulation.

In [9] Wilks' Lambda is used for change detection in both simulated data and quadpol SAR data.

\section{THEORY}

Consider two $p \times p$ independent, Hermitian, positive definite variance-covariance matrices $\boldsymbol{X}$ and $\boldsymbol{Y}$ representing geometrically co-registered multi-look covariance SAR data at two time points, $\boldsymbol{X}$ at $t_{1}$ and $\boldsymbol{Y}$ at $t_{2}, t_{2}>t_{1}$.

Below we confine ourselves to dual or single polarization, and we look at the real case where we have diagonal elements of the covariance matrix or single channel intensity or power data only.

A. A. Nielsen is an emeritus with DTU Compute, the Department of Applied Mathematics and Computer Science, Technical University of Denmark, DK-2800 Kgs. Lyngby, Denmark. e-mail alan@dtu.dk, homepage https://people.compute.dtu.dk/alan.

\section{A. Wilks' Lambda}

Wilks' Lambda [8] is defined as the ratio of the determinants

$$
\Lambda=\frac{|\boldsymbol{X}|}{|\boldsymbol{X}+\boldsymbol{Y}|},
$$

$\Lambda \in[0,1]$. If $\Lambda$ is large, $\boldsymbol{X}$ dominates the ratio and in a change detection setting "something is removed" at time point two ("something" being radar signal as expressed by the determinant of the covariance matrix). If on the other hand $\Lambda$ is small, $\boldsymbol{Y}$ dominates and "something is added" at time point two.

$\boldsymbol{X}=\boldsymbol{Y}$ depicts no change between the two time points. This leads to $\Lambda=2^{-p}$, i.e., $1 / 4$ for dual polarization $(p=2)$, and $1 / 2$ for single channel power data $(p=1)$.

Alternatively, we could have $|\boldsymbol{Y}|$ in the numerator in (1). Due to the symmetry in the handling of data from the two time points, analysis where we look at both the lower and upper tails of the distributions of both $|\boldsymbol{X}| /|\boldsymbol{X}+\boldsymbol{Y}|$ and $|\boldsymbol{Y}| /|\boldsymbol{X}+\boldsymbol{Y}|$ is preferred over looking at lower and upper tails of $|\boldsymbol{X}| /|\boldsymbol{X}+\boldsymbol{Y}|$ only.

\section{B. Distribution of Wilks' Lambda}

Wilks' $\Lambda$ also known as Anderson's $U$ [8] for the so-called null hypothesis $H_{0}: \boldsymbol{X}=\boldsymbol{Y}$ (i.e., no change) versus the alternative hypothesis $H_{1}: \boldsymbol{X} \neq \boldsymbol{Y}$, follows a complicated three parameter distribution. For data following the real (as opposed to the complex) Wishart distribution, often used approximations for Wilks' $\Lambda$ are based on the $\chi^{2}$ (chi-squared) distribution or the $F$ distribution. Here, we follow another line of attack based on simulations of outcomes of the (complex) Wishart distribution and a Beta distribution approximation to Wilks' $\Lambda$ rather than theoretical derivation.

Specifically, to emulate a no change situation in a change detection setting, we generate two times $1024^{2}$ observations following the (complex) Wishart distribution (see [10], [11]) with the same parameters, only we vary the number of looks. To emulate Sentinel- ${ }^{1}$ data acquired from the Google Earth Engine $^{2}$ (GEE) [12]), we do this for dual polarization diagonal only data simply by removing the simulated off-diagonal complex elements.

The Beta distribution is a good approximation to many continuous distributions on the interval $[0,1]$ such as probabilities. For positive $\alpha$ and $\beta$ the probability density function is

$$
f(x)=\frac{x^{\alpha-1}(1-x)^{\beta-1}}{B(\alpha, \beta)}, 0<x<1
$$

\footnotetext{
${ }^{1} \mathrm{https} / / /$ sentinel.esa.int/web/sentinel/missions/sentinel-1

${ }^{2}$ https://earthengine.google.com
} 


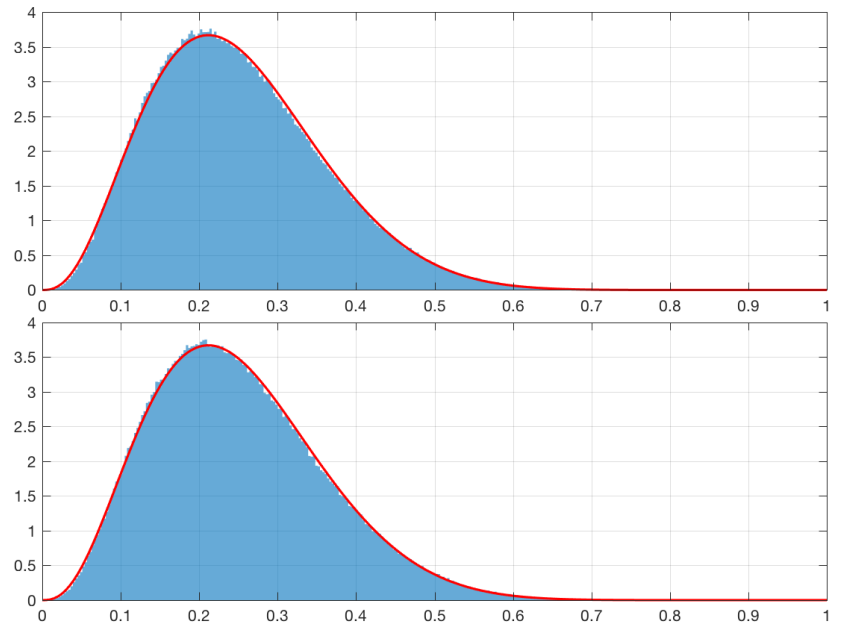

Fig. 1. Histograms (in blue) for Wilks' $\Lambda(|\boldsymbol{X}| /|\boldsymbol{X}+\boldsymbol{Y}|$ top and $|\boldsymbol{Y}| / \mid \boldsymbol{X}+$ $\boldsymbol{Y} \mid$ bottom) and fitted Beta distribution (in red) for generated no change data, dual polarization diagonal only, $L=5$.

and $f(x)=0$ elsewhere. To indicate that a stochastic variable $X$ follows a Beta distribution, we write $X \sim B e(\alpha, \beta)$. Above $B(\alpha, \beta)$ is the Beta function

$$
\begin{aligned}
B(\alpha, \beta) & =\int_{0}^{1} t^{\alpha-1}(1-t)^{\beta-1} d t \\
& =\frac{\Gamma(\alpha) \Gamma(\beta)}{\Gamma(\alpha+\beta)}, \alpha>0, \beta>0 .
\end{aligned}
$$

Here $\Gamma(\alpha)$ is the Gamma function

$$
\Gamma(\alpha)=\int_{0}^{\infty} t^{\alpha-1} \mathrm{e}^{-t} d t, \alpha>0,
$$

$\Gamma(1)=1$ and $\alpha \Gamma(\alpha)=\Gamma(\alpha+1)$, hence for integer numbers $\Gamma(n)=(n-1)$ !, i.e., $\Gamma(n)$ is $n-1$ factorial.

We now fit Beta distributions to the histograms obtained for Wilks' Lambda as a function of the number of looks, $L=$ $2,3,4,5,6,10,20,30,100,170,180,190,194,195,196,197$,

198. This is done by means of maximum likelihood estimation as implemented in Matlab function fitdist. Figures 1 and 2 show two examples for $L=5$ (the nearest integer to the equivalent number of looks (ENL) of 4.9 given earlier by ESA for the Sentinel-1 data used below, now that number is 4.4), and for $L=20$. Both examples shown (as well as examples not shown), exhibit good agreement with the sample histograms (judged visually, there is a tendency to a slightly better fit for higher $L$ ).

Following this, we fit straight lines to the estimates obtained for $\alpha$ and $\beta$ as functions of $L$. Figure 3 shows the functions for dual polarization diagonal only data. The estimated values are $\alpha=0.750 L$ and $\beta=2.250 L$, see tables below (we get highly insignificant estimates for the intercepts which are therefore removed).

\begin{tabular}{|l|rrcc|}
\hline \hline$\alpha$ & Est. & Std. err. & $t$ & $P\{>|t|\}$ \\
\hline Intercept & -0.00662 & 0.04413 & -0.1500 & 0.8828 \\
Slope & 0.75019 & 0.000332 & 2262.0 & $6.510^{-43}$ \\
\hline Slope & 0.75015 & 0.000212 & 3538.5 & $1.410^{-48}$ \\
\hline \hline
\end{tabular}

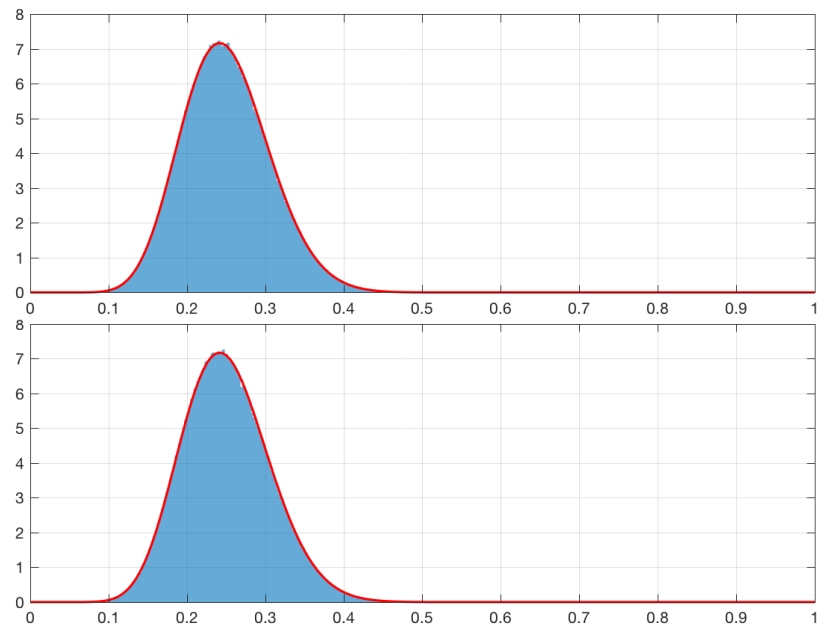

Fig. 2. Histograms (in blue) for Wilks' $\Lambda(|\boldsymbol{X}| /|\boldsymbol{X}+\boldsymbol{Y}|$ top and $|\boldsymbol{Y}| / \mid \boldsymbol{X}+$ $\boldsymbol{Y} \mid$ bottom) and fitted Beta distribution (in red) for generated no change data, dual polarization diagonal only, $L=20$.

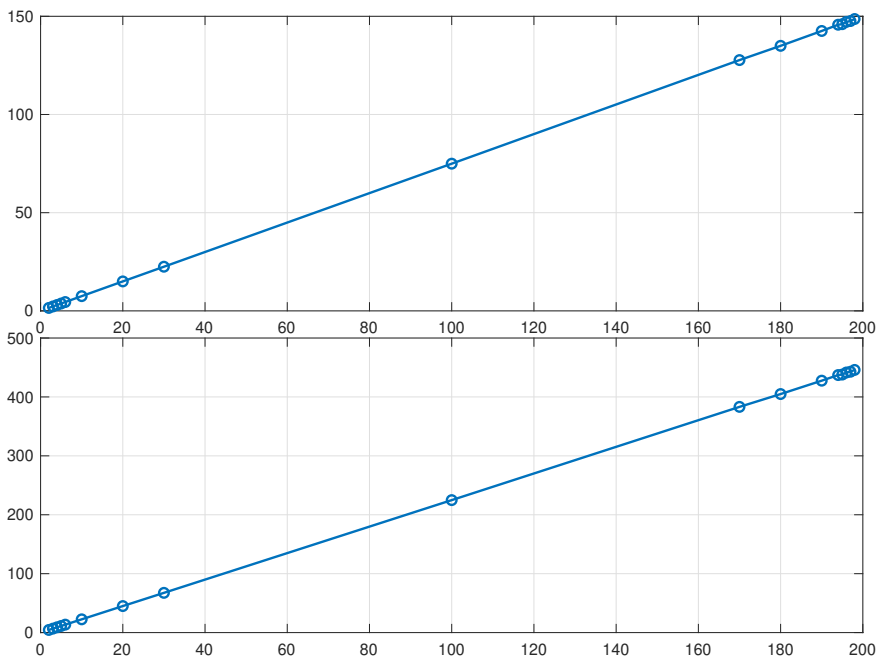

Fig. 3. Linear fits for $\alpha$ (top) and $\beta$ (bottom) as functions of $L$ for generated no change data, dual polarization diagonal only.

\begin{tabular}{|l|rrcc|}
\hline \hline$\beta$ & Est. & Std. err. & $t$ & $P\{>|t|\}$ \\
\hline Intercept & -0.02896 & 0.13267 & -0.2183 & 0.8302 \\
Slope & 2.2506 & 0.000997 & 2257.3 & $6.710^{-43}$ \\
\hline Slope & 2.2505 & 0.000638 & 3528.1 & $1.510^{-48}$ \\
\hline \hline
\end{tabular}

Residual analysis shows that the absolute values of the residuals generally increase with $L$ and that no standardized residual is above 2 or below -3 . The leverage of observations increases with $L$, no leverage value is too high.

Similar regression analysis for single polarization data results in estimates $\alpha=\beta=L$.

It is stressed that this simulation study including the regression models was performed under the null hypothesis that there is no change between acquisitions times.

\section{Case Study On Sentinel-1 Data}

Our case study is based on dual polarization (VV and VH) Sentinel-1 C-band data over the international Frankfurt 

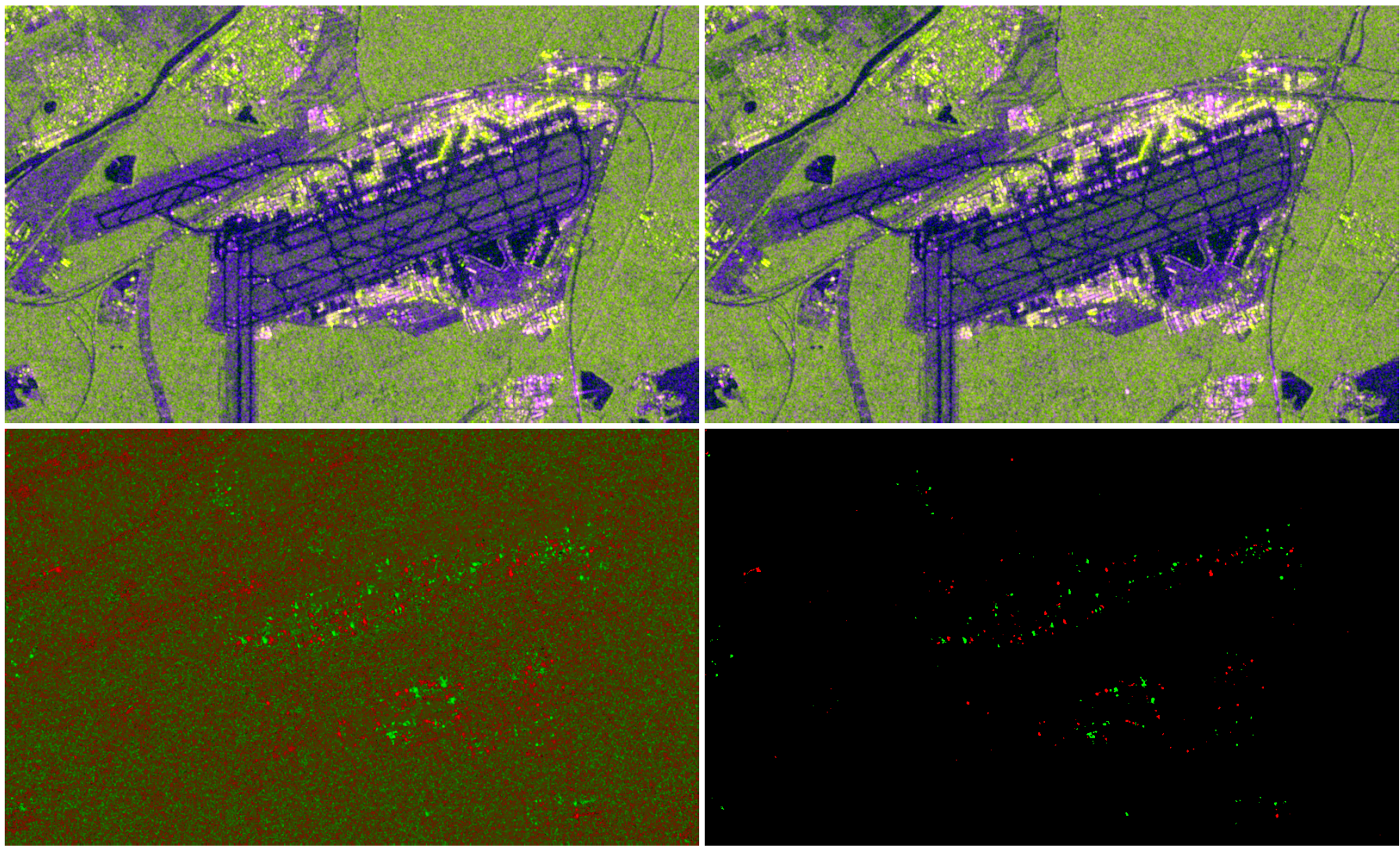

Fig. 4. Sentinel-1 $S_{v v} S_{v v}^{*}, S_{v h} S_{v h}^{*}$ and the ratio $S_{v v} S_{v v}^{*} /\left(S_{v h} S_{v h}^{*}\right)$ for the two time points as RGB (top row), Wilks' $\Lambda|\boldsymbol{X}| /|\boldsymbol{X}+\boldsymbol{Y}|$ in red and $|\boldsymbol{Y}| /|\boldsymbol{X}+\boldsymbol{Y}|$ in green (bottom row left), and the same combination, both tails thresholded at $0.005 \%$ and $99.995 \%$ (bottom row right).

Airport, Germany.

The 4.9-look images used are 600 rows by 1000 columns $10 \mathrm{~m}$ pixels acquired in IW mode on 29 March and 10 April 2016. The data (VV and VH only, no off-diagonal elements in the covariance matrix) are obtained from and preprocessed by the Google Earth Engine (GEE) [12].

Figure 4 shows $S_{v v} S_{v v}^{*}, \quad S_{v h} S_{v h}^{*}$ and the ratio $S_{v v} S_{v v}^{*} /\left(S_{v h} S_{v h}^{*}\right)$ for the two time points as RGB (top row), Wilks' Lambda $|\boldsymbol{X}| /|\boldsymbol{X}+\boldsymbol{Y}|$ in red and $|\boldsymbol{Y}| /|\boldsymbol{X}+\boldsymbol{Y}|$ in green (bottom row left), and the same combination with both lower and upper tails of the estimated Beta distribution thresholded at $0.005 \%$ and $99.995 \%$ (bottom row right).

Where the bottom row images are red, time point one dominates the ratio and "something is removed" at time point two, where they are green, time point two dominates the ratio and "something is added" at time point two.

Figure 5 shows Wilks' $\Lambda$ from a part of Figure 4 bottom row right overlaid in Google Earth ${ }^{3}$.

We see mostly aircraft and vehicles coming and going at the gates, on the aprons, taxiways and runways, and on the motorway, as well as ships coming and going on the River Main.

The results shown in Figures 4 and 5 are very similar to results from detection by means of the Wishart test statistic [1], [2] followed by determination of the Loewner order of the difference image $\boldsymbol{X}-\boldsymbol{Y}$ shown in Figure 6, see [13],

${ }^{3}$ https://earth.google.com
[14] where the latter reference gives computational speed-up of factors in the hundreds of the methods described in the former. The Loewner order determines whether the difference matrix is positive definite, negative definite or indefinite. Hence, it gives a measure of direction of detected change. In the few situations where the combined Wishart-Loewner method detects significant change with indefinite difference matrices shown in yellow in Figure 6, Wilks' Lambda in this example appears to detect no change. This indicates that the combined Wishart-Loewner measure is richer in change information content than Wilks' Lambda.

No change from time point one to time point two, $\boldsymbol{X}=\boldsymbol{Y}$, in this case ( $p=2$ and $m=n=4.9$ ) leads to mean value 0.25 for Wilks' Lambda with $\alpha=3.6750$ and $\beta=11.0250$ for the approximate Beta distribution. Figure 7 shows histograms of Wilks' Lambda, $|\boldsymbol{X}| /|\boldsymbol{X}+\boldsymbol{Y}|$ (top) and $|\boldsymbol{Y}| /|\boldsymbol{X}+\boldsymbol{Y}|$ (bottom), for the Sentinel-1 data in a wooded no change region immediately west of the runways. Judged visually the sample histograms nicely follow the estimated Beta distribution. More formally, the Kolmogorov-Smirnov test statistic for the null hypothesis that the sample histogram and the corresponding values from the Beta distribution are from the same continuous (Beta) distribution is 0.1667 with p-value 0.7601 for $|\boldsymbol{X}| /|\boldsymbol{X}+\boldsymbol{Y}|$. For $|\boldsymbol{Y}| /|\boldsymbol{X}+\boldsymbol{Y}|$ the Kolmogorov-Smirnov test statistic is 0.1333 with p-value 0.9360 . In both cases we cannot reject the null hypothesis: for this no-change region the fits to the approximated theoretical distribution are good. 


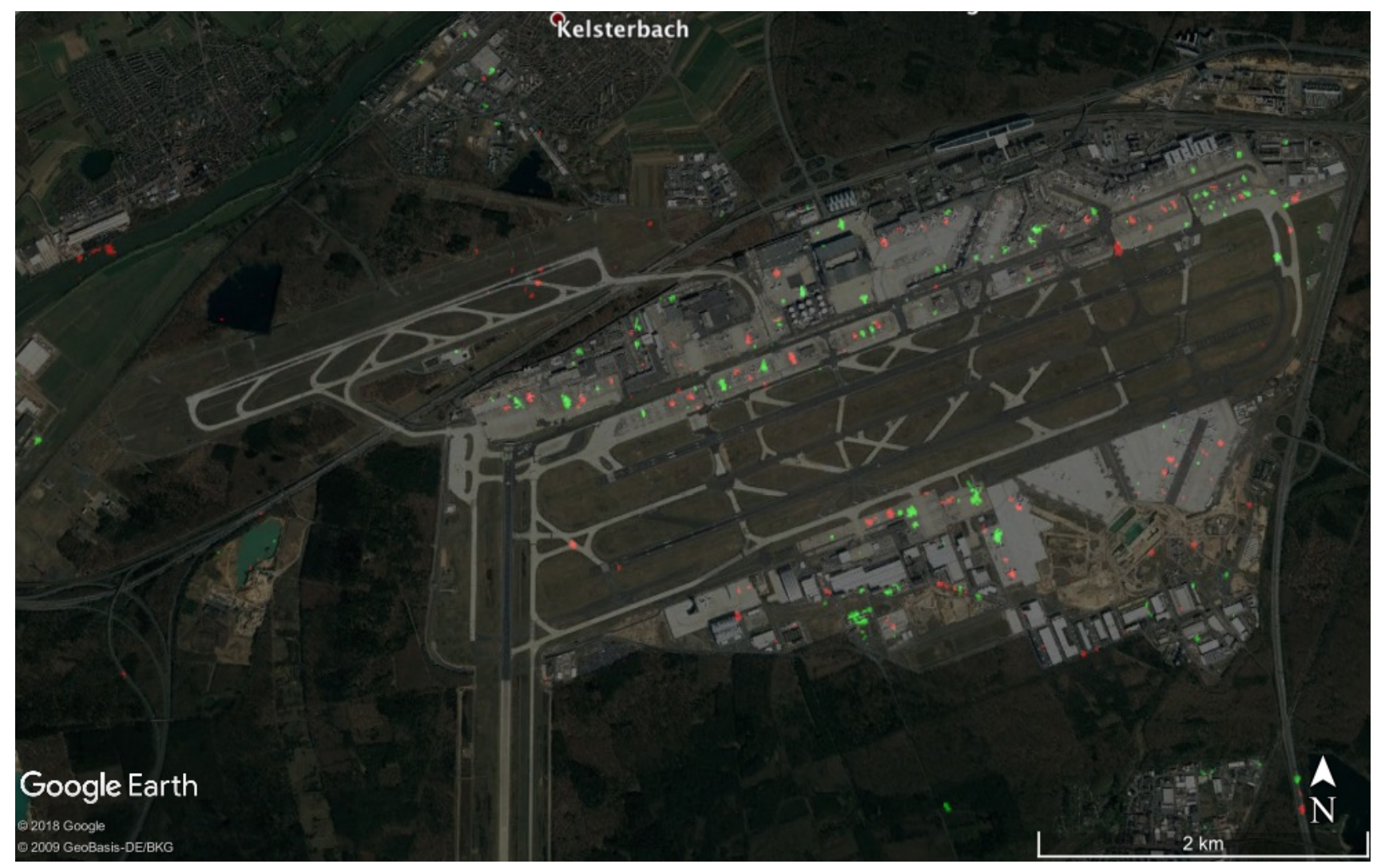

Fig. 5. Wilks' $\Lambda$ from a part of Figure 4 bottom row right overlaid in Google Earth.

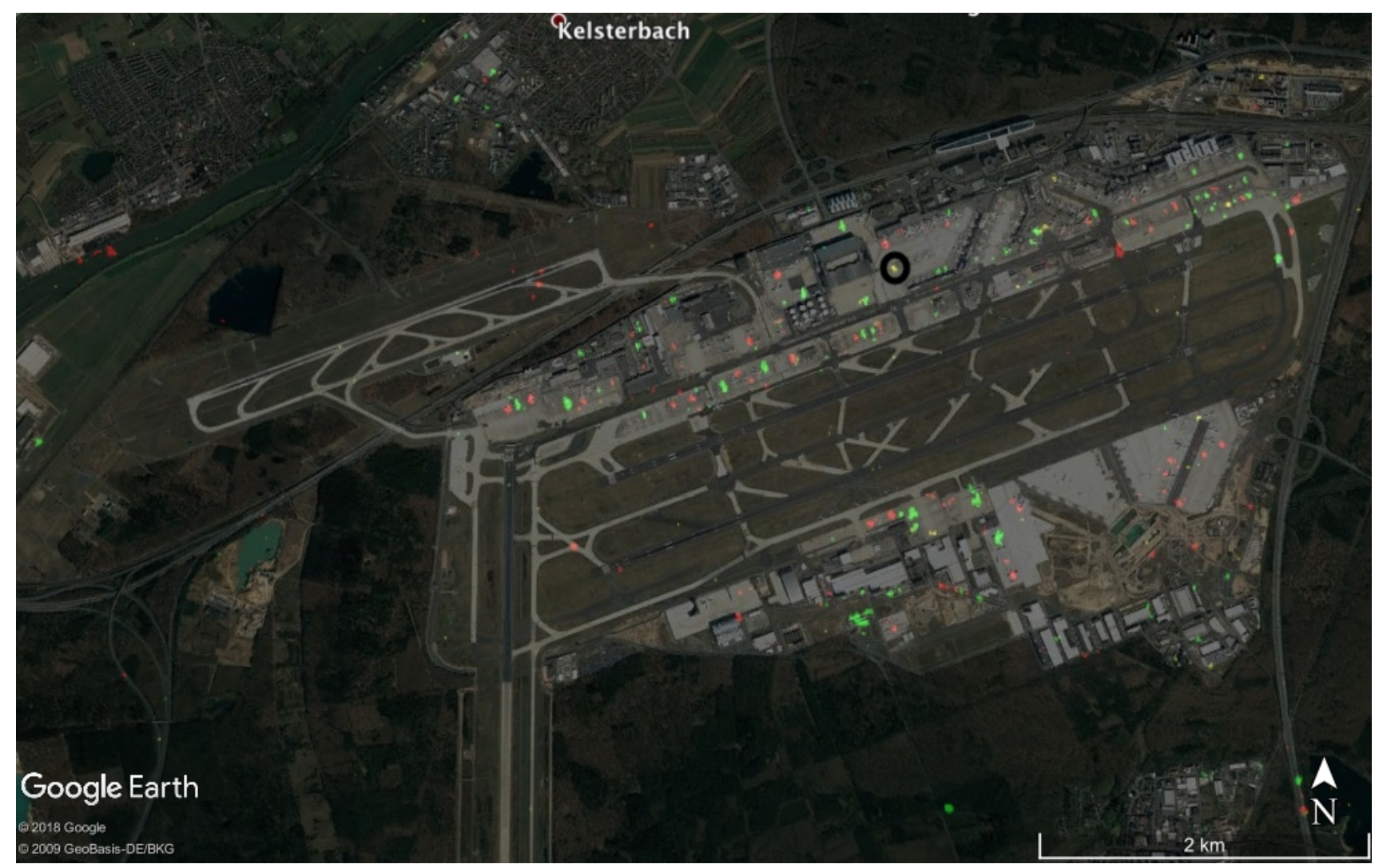

Fig. 6. Result from the combined Wishart-Loewner method described in [13] based on the same data covering the same area as in Figure 5 overlaid in Google Earth. A few indefinite matrix differences (in yellow) are seen, an example on the north side of the airport is marked with a black circle. These statistically significant changes are not detected by Wilks' $\Lambda$ indicating that the combined Wishart-Loewner measure is richer in change information content. 

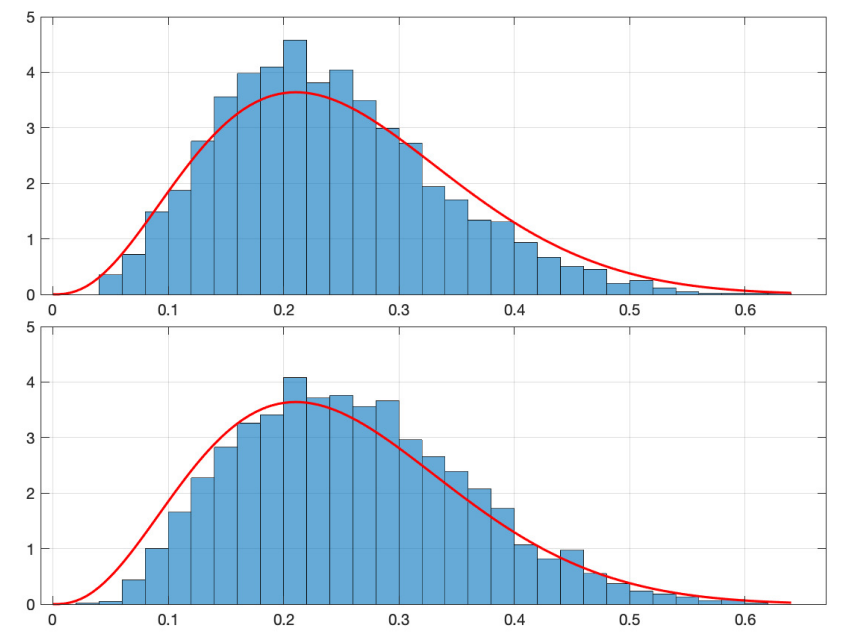

Fig. 7. Histograms for Wilks' Lambda $|\boldsymbol{X}| /|\boldsymbol{X}+\boldsymbol{Y}|$ (top) and $|\boldsymbol{Y}| / \mid \boldsymbol{X}+$ $\boldsymbol{Y} \mid$ (bottom), and Beta distribution approximation $(\alpha=3.6750$ and $\beta=$ 11.0250), Sentinel-1 data, dual polarization diagonal only in a wooded no change region immediately west of the runways.

\section{CONCLUSIONS}

Unlike other methods for change detection in polarimetric SAR data in the covariance matrix representation in [1][4], [6], and most methods published by other authors, this paper gives a method that can give the direction of change by determining whether the radar signal (as measured by the determinant of the covariance matrix) has increased or decreased over time.

It is emphasized that the simulation study including the regression models was performed under the null hypothesis of no change between acquisitions times.

In an example with Sentinel-1 dual polarization diagonal only data, change is succesfully detected by Wilks' Lambda and an associated p-value from a simulation based Beta distribution approximation.

For a no-change region fits to the approximated theoretical distribution are good judged both visually and more formally by means of the Kolmogorov-Smirnov test.

Detection results are very similar to results from detection by means of the Wishart test statistic [1]-[4], [6], followed by determination of the Loewner order of the difference image as described in [13]. However, in the few situations where the combined Wishart-Loewner measure detects significant change with indefinite difference matrices, Wilks' Lambda here tends to detect no change. This indicates that the combined WishartLoewner measure is richer in change information content than Wilks' Lambda.

Matlab code wilks_lambda_change.m to perform the analysis is available on the author's homepage.

\section{ACKNOWLEDGEMENT}

The author would like to thank an anonymous reviewer for comments which helped improve especially the regression analysis part of the original manuscript.

\section{REFERENCES}

[1] K. Conradsen, A. A. Nielsen, J. Schou, and H. Skriver, "A test statistic in the complex Wishart distribution and its application to change detection in polarimetric SAR data," IEEE Transactions on Geoscience and Remote Sensing, vol. 41, no. 1, pp. 4-19, Jan. 2003, https://doi.org/10.1109/TGRS.2002.808066.

[2] M. J. Canty, Image Analysis, Classification and Change Detection in Remote Sensing, with Algorithms for Python, Taylor \& Francis, CRC Press, fourth revised edition, 2019.

[3] A. A. Nielsen, K. Conradsen, and H. Skriver, "Change detection in full and dual polarization, single- and multi-frequency SAR data," IEEE Journal of Selected Topics in Applied Earth Observations and Remote Sensing, vol. 8, no. 8, pp. 4041-4048, Aug. 2015, https://doi.org/10.1109/JSTARS.2015.2416434.

[4] K. Conradsen, A. A. Nielsen, and H. Skriver, "Determining the points of change in time series of polarimetric SAR data," IEEE Transactions on Geoscience and Remote Sensing, vol. 54, no. 5, pp. 3007-3024, May 2016, https://doi.org/10.1109/TGRS.2015.2510160.

[5] V. Akbari, S. N. Anfinsen, A. P. Doulgeris, T. Eltoft, G. Moser, and S. B. Serpico, "Polarimetric SAR change detection with the complex Hotelling-Lawley trace statistic," IEEE Transactions on Geoscience and Remote Sensing, vol. 54, no. 7, pp. 3953-3966, Jul. 2016, https://doi.org/10.1109/TGRS.2016.2532320.

[6] A. A. Nielsen, K. Conradsen, H. Skriver, and M. J. Canty, "Visualization of and software for omnibus test based change detected in a time series of polarimetric SAR data," Canadian Journal of Remote Sensing, vol. 43, no. 6, pp. 582-592, 2017, https://doi.org/10.1080/07038992.2017.1394182.

[7] A. D. C. Nascimento, A. C. Frery, and R. J. Cintra, "Detecting changes in fully polarimetric SAR imagery with statistical information theory," IEEE Transactions on Geoscience and Remote Sensing, vol. 57, no. 3, pp. 1380-1392, Mar. 2019, https://doi.org/10.1109/TGRS.2018.2866367.

[8] T. W. Anderson, An Introduction to Multivariate Statistical Analysis, John Wiley, New York, third edition, 2003.

[9] N. Bouhlel, V. Akbari, and S. Méric, "Change detection in multilook polarimetric SAR imagery with determinant ratio test stastitic," IEEE Transactions on Geoscience and Remote Sensing (Early Access), Dec. 2020, https://doi.org/10.1109/TGRS.2020.3043517.

[10] P. L. Odell and A. H. Feiveson, "A numerical procedure to generate a sample covariance matrix," Journal of the American Statistical Association, vol. 61, no. 313, pp. 199-203, 1966, https://doi.org/10.1080/01621459.1966.10502018.

[11] A. A. Nielsen, H. Skriver, and K. Conradsen, "Generation of sample complex Wishart distributed matrices and change detection in polarimetric SAR data," in International Conference on Digital Image and Signal Processing (DISP), Oxford, England, Apr. 2019, http://www2.imm.dtu.dk/pubdb/p.php?7124.

[12] N. Gorelick, M. Hancher, M. Dixon, S. Ilyushchenko, D. Tau, and R. Moore, "Google Earth Engine: Planetary-scale geospatial analysis for everyone," Remote Sensing of Environment, vol. 202, pp. 18-27, 2017, https://doi.org/10.1016/j.rse.2017.06.031.

[13] A. A. Nielsen, H. Skriver, and K. Conradsen, "The Loewner order and direction of detected change in Sentinel-1 and Radarsat-2 data," IEEE Geoscience and Remote Sensing Letters, vol. 17, no. 2, pp. 242-246, Feb. 2020, https://doi.org/10.1109/LGRS.2019.2918636.

[14] A. A. Nielsen, "Fast matrix based computation of eigenvalues and the Loewner order in polSAR data," IEEE Geoscience and Remote Sensing Letters, vol. 17, no. 10, pp. 1727-1731, Oct. 2020, https://doi.org/10.1109/LGRS.2019.2952202. 